Pacific Journal of Mathematics

FUNCTIONS OF TRANSLATION TYPE AND SOLID BANACH 


\title{
FUNCTIONS OF TRANSLATION TYPE AND SOLID BANACH SPACES OF FUNCTIONS
}

\author{
REINHARD BÜRGER
}

Functions of translation type were introduced by H. Reiter and studied by the author in some detail. In this paper we introduce a class of Banach spaces of functions on a locally compact group, including the spaces $\mathscr{L}^{p}(G)$ of Liu-van Rooij-Wang. Necessary and sufficient conditions are given under which these spaces can be characterized by functions of translation type. As application it is shown that a subclass of these spaces, including Wiener's algebra, satisfies a certain minimality property. Furthermore, we obtain a generalization of a theorem on Fourier transforms, due to Edwards-Hewitt-Ritter, in a very simple manner, whereas the original proof took several pages.

Our notation follows that of [13]. G always denotes an arbitrary locally compact group with left Haar measure $d x$. For a measurable set $M \subset G$ let $|M|_{G}$ be the left Haar measure of $M$. $\Delta_{G}$ denotes the Haar modulus of $G$.

We write $\mathscr{C}(G)$ for the linear space of all continuous, complexvalued functions on $G, \mathscr{C}^{\circ}(G)$ for the Banach space of all continuous functions vanishing at infinity with the norm $\|f\|_{\infty}=\sup _{x \in G}|f(x)|$ and $\mathscr{K}(G)$ for the subspace of all functions with compact support (supp). $L_{\mathrm{ioc}}^{1}(G)$ denotes the space of all locally integrable functions on $G$. As usual measurable functions coinciding locally almost everywhere (l.a.e.) shall be identified. The spaces $L^{p}(G)$ have the usual meaning. The left [right] translation operators $L_{y}\left[R_{y}\right]$ are defined by $L_{y} f(x):=f\left(y^{-1} x\right)\left[R_{y} f(x):=f(x y)\right]$. We call two functions $w_{1}$ and $w_{2}$ equivalent (l.a.e.), $w_{1} \sim w_{2}$ (l.a.e.) if there exist constants $C_{1}, C_{2}>0$ such that $C_{1} w_{1}(x) \leqq w_{2}(x) \leqq C_{2} w_{1}(x)$ (l.a.e.). $\Phi_{A}$ shall denote the characteristic function of the set $A$. Given a measurable, locally essentially bounded function $w$ on $G,\left(L_{w}^{1}(G),\|\|_{1, w}\right)$ denotes the space of all measurable functions $f$ on $G$ such that $f w$ is in $L^{1}(G)$ with the norm $\|f\|_{1, w}:=\|f w\|_{1}$. This space is a Banach convolution algebra if and only if $w \sim w_{1}$ l.a.e. for some $w_{1} \in L_{10 \mathrm{co}}^{1}(G)$ satisfying $0<w_{1}<\infty$ and $w_{1}(x y) \leqq C w_{1}(x) w_{1}(y)$ for all $x, y \in G$ (cf. [9]). Moreover, $L_{w}^{1}(G)$ possesses bounded approximate left units since $\mathscr{K}(G)$ is dense in $L_{w}^{1}(G)$.

Throughout this paper we shall always consider Banach spaces $\left(B(G),\|\|_{B}\right)$ of measurable functions, continuously embedded into $L_{\text {loc }}^{1}(G)$ and satisfying the following conditions:

(B1) $B(G)$ is left invariant, i.e., $L_{y}$ defines a continuous linear operator on $B(G)$ for every $y \in G$. 
(B2) $B(G)$ is a solid Banach space of [continuous] functions, i.e., $f \in B(G)$ and $|g(x)| \leqq|f(x)|$ l.a.e. for any measurable [continuous] function $g$ implies $g \in B(G)$ and $\|g\|_{B} \leqq\|f\|_{B}$.

(B3) $B(G)$ is a Banach convolution module over some Beurling algebra $L_{w}^{1}(G)$, i.e., $h \in L_{w}^{1}(G)$ and $f \in B(G)$ implies $h * f \in B(G)$ and $\|h * f\|_{B} \leqq\|h\|_{1, w}\|f\|_{B}$.

REMARK 0.1. (i) Each Banach function space satisfying (B1) (B3) contains $\mathscr{K}(G)$ as a subspace (cf. [6]).

(ii) If $\mathscr{K}(G)$ is dense in $B(G)$ then the map $y \rightarrow L_{y} f$ is continuous for each $y \in G$ and $f \in B(G)$ (cf. [6]).

(iii) The continuity of the map $y \rightarrow L_{y} f$ is equivalent to the fact that $B(G)$ satisfies (B3) with $w(y):=\max \left(1,\left\|L_{y}\right\|_{B}\right)$ and that $L_{w}^{1}(G) * B(G)$ is dense in $B(G)$ (cf. [7]). Thus, if especially $\left\|L_{y}\right\|_{B}=1$ for all $y \in G, B(G)$ is an essential Banach convolution module over $L^{1}(G)$.

(iv) If there is a constant $C$ such that $\left\|L_{y}\right\|_{B} \leqq C$ holds for all $y \in G$ one can choose an equivalent norm \|\|$_{B}^{\prime}$ on $B(G)$ such that $\left\|L_{y}\right\|_{B}^{\prime}=1$ for all $y \in G$, in particular we have $\left\|L_{y} f\right\|_{B}^{\prime}=\|f\|_{B}^{\prime}$ for all $f \in B(G)$.

I. Functions of translation type. Basic results. Functions of translation type were introduced by $H$. Reiter in [14] to show certain functorial properties of the space $\mathfrak{S}(G)$ of Schwartz-Bruhat functions. Their properties are discussed in some detail in [2] and [3]. Let us shortly recall the definition and derive some results that will be used in the proof of our main result.

Definition 1.1. A continuous real-valued function $\omega: G \rightarrow R$, $\omega \geqq 0$, is called a function of [left] translation type if it satisfies the following condition $(\mathrm{V})\left[\left(V_{L}\right)\right]$ :

There is an open, compactly generated subgroup $G(\omega)<G$ with the property that for each compact set $K \subset G(\omega)$ there exists a constant $C_{K}>0$ such that $\omega(x a) \leqq C_{K} \omega(x)\left[\omega\left(a^{-1} x\right) \leqq C_{K} \omega(x)\right]$ holds for all $x \in G$ and each $a \in K$.

By $V(G)\left[V_{L}(G)\right]$ we shall denote the cone of all functions of [left] translation type. Furthermore, we put $V^{B}(G):=V(G) \cap B(G)$ and we write $V^{p}(G)$ and $V^{1, w}(G)$ if $B(G)=L^{p}(G)$ or $L_{w}^{1}(G)$ (analogously $V_{L}^{p}(G$ ) and $V_{L}^{1, w}(G)$ ). Moreover, we denote $V^{B}(G) \cap V_{L}^{B}(G)$ by $V_{T}^{B}(G)$.

REMARK 1.1. (i) Each $\omega \in V^{B}(G)$ has a representation of the form $\omega=\sum_{n \geq 1} L_{y_{n}} \omega_{n}$ with $y_{n} \in G$ and $\omega_{n} \in V^{B}(G), \omega_{n}(x) \neq 0 \Leftrightarrow x \in G(\omega)$.

(ii) If $G$ is connected one has to choose $G(\omega)=G$.

(iii) A discrete group $G$ is not of interest for the considerations 
of this paper, since in this case we may choose $G(\omega)=\{e\}$ and hence $V_{T}^{B}(G)=B(G)$.

LEMMA 1.1. Let $\omega \in L^{1}(G), \omega \geqq 0$, be given such that $\omega$ satisfies (V) $\left[\left(V_{L}\right)\right]$. If $k \in \mathscr{K}_{+}(G)$ such that supp $k \subset G(\omega)$ then $\omega \sim \omega * k$ and $\omega * k \in V^{1}(G)\left[V_{L}^{1}(G)\right]$.

Proof. We shall only consider the case (V) since the other case is even easier.

It is obvious that $\omega * k$ is a continuous, positive function in $L^{\prime}(G)$. If we denote $K:=\operatorname{supp} k$, we get:

$$
\begin{aligned}
\omega * k(x) & =\int_{K} \Delta_{G}\left(y^{-1}\right) \omega\left(x y^{-1}\right) k(y) d y \leqq C_{K^{-1}} \cdot \int_{K} \Delta_{G}\left(y^{-1}\right) k(y) d y \cdot \omega(x) \\
& =C_{1} \omega(x)
\end{aligned}
$$

and

$$
\begin{aligned}
\omega(x) & =C^{\prime} \cdot \int_{K} \Delta_{G}\left(y^{-1}\right) k(y) \omega(x) d y \leqq C^{\prime} \cdot C_{K} \cdot \int_{K} \Delta_{G}\left(y^{-1}\right) \omega\left(x y^{-1}\right) k(y) d y \\
& =C_{2} \cdot \omega * k(x)
\end{aligned}
$$

and hence $\omega * k \in V^{j}(G)$.

LEMMA 1.2. Let $\rho$ be a locally essentially bounded function on $G$ satisfying $\rho \geqq 0$ and $\rho(x y) \leqq \rho(x) \rho(y)$. Then for every function $\omega_{1} \in V_{T}^{1}(G)$ we can find some $\omega \in V_{T}^{1}(G)$ such that $\omega \cdot \rho \leqq \omega_{1}$ holds.

Proof. Let $V \subset G$ be a symmetric, compact neighbourhood of $e$ and let $G^{\prime}$ be the open subgroup of $G$ generated by $V$. Then there exists a constant $p>1$ such that $\left|K^{n}\right|_{G} \leqq|K|_{G} \cdot p^{n}, n \in N$ (cf. [11]). Moreover, the existence of a constant $q>0$ satisfying

$$
\sup \left\{\rho(x) \mid x \in V^{n}\right\} \leqq q^{n}
$$

follows from the assumptions imposed on $\rho$. If we choose now a constant $a>\max \{p, q\}$ the function $g(x):=\sum_{n=1}^{\infty} a^{-n} \Phi_{V^{n} \backslash V^{n-1}}(x)$ satisfies $g\left(y^{-1} x\right) \leqq a g(x)$ as well as $g(x y) \leqq a g(x)$ for all $x \in G$ and each $y \in V$ and hence the properties $(V)$ and $\left(V_{L}\right)$ are satisfied for $G(g)=G^{\prime}$. Moreover, it is obvious that $g(x) \rho(x) \leqq 1$ holds for all $x \in G$. Using Lemma 1.1 we get for any $k \in \mathscr{K}_{+}(G)$ with supp $k \subset G^{\prime}: g * k \sim g$ and $g * t \in V_{T}^{1}(G)$. If we choose now $\omega_{1} \in V_{T}^{1}(G)$ and put $\omega:=\omega_{1} \cdot(g * k)$ we derive $\omega \cdot \rho \leqq \omega_{1} \cdot(g * k) \cdot \rho \leqq C^{\prime} \cdot \omega_{1} \cdot g \cdot \rho \leqq C^{\prime} \cdot \omega_{1}$. Since $\omega \in V_{T}^{1}(G)$ the proof is complete.

CoRollary 1.3. Let $G$ be an arbitrary locally compact group. 
Then we have: $V_{T}^{B}(G) \neq\{0\}$.

Proof. Due to Lemma 1.2 there exists a nontrivial function $\omega \in V^{1, w}(G)$. Hence, by Lemma $1.1 \omega * k \in V^{B}(G)$ for each $k \in \mathscr{K}_{+}(G) \subset$ $B(G)$ with supp $k \subset G(\omega)$.

LEMma 1.4. If $\mathscr{K}(G)$ is dense in $B(G)$ then $V^{B}(G)$ is dense in $B_{+}(G)$.

Proof. For every $f \in B_{+}(G)$ there exists some $k \in \mathscr{K}_{+}(G)$ such that $\|f-k\|_{B}<\varepsilon / 2$. Moreover, choose some $\omega \in V^{B}(G)$ with $\|\omega\|_{B}<$ $\varepsilon / 2$ and $\operatorname{supp} k \subset \operatorname{supp} \omega$. Then it is easily seen that $\omega^{\prime}:=\omega+k \epsilon$ $V^{B}(G)$ and hence $\left\|f-\omega^{\prime}\right\|_{B}<\varepsilon$.

REMARK 1.2. (i) The left version of Lemma 1.2 will be used in a crucial way in order to prove our main theorem.

(ii) $V^{1, w}(G)$ is dense in $\left(L_{w}^{1}\right)_{+}(G)$.

(iii) If $\mathscr{K}(G)$ is dense in $B(G)$ and $\left\|L_{y}\right\|_{B}=1$ for all $y \in G$ then $V^{B}(G)$ is contained in $\mathscr{C}^{\circ}(G)$. Since the proof is essentially the same as the proof of Proposition 1 of [2] it is omitted.

II. The main results, Let us begin with the following definition:

Definition 2.1. (i) $\mathfrak{B}^{B}(G):=\left\{f \in \mathscr{C}(G) \mid\right.$ there exists some $\omega_{f} \in$ $V^{B}(G)$ with $\left.|f| \leqq \omega_{f}\right\}$.

(ii) Let $g \in \mathscr{K}(G), g \neq 0$, and put $f^{g}(x):=\left\|\left(L_{x} g\right) \cdot f\right\|_{\infty}$. Then we define: $\mathscr{C}_{g}^{B}(G):=\left\{f \in \mathscr{C}(G) \mid f^{g} \in B(G)\right\}$.

(iii) $B^{g}(G):=\left\{f^{g} \mid f \in \mathscr{A}_{g}^{B}(G)\right\}$.

If $B=L^{p}$ we shall write $\mathfrak{B}^{p}(G)$ and $\mathscr{L}_{g}^{p}(G)$. If $B=L_{w}^{1}$ we write $\mathfrak{B}^{1, w}(G)$ and $\mathscr{L}_{g}^{1, w}(G)$.

THeOREM 2.1. $\mathscr{C l}_{g}^{B}(G)$ is a Banach space with the norm $\|f\|_{(B)}:=$ $\left\|f^{g}\right\|_{B}$ that is continuously embedded into $B(G)$ and satisfies (B1)-(B3) with respect to each Banach algebra $L_{w}^{1}(G)$ that acts on $B(G)$.

Proof. It is obvious that \|\|$_{(B)}$ is a norm and that $\mathscr{L}_{g}{ }^{B}(G)$ is a Banach space with this norm, continuously embedded into $B(G)$ and satisfying (B1) and (B2). To show (B3) let $h \in L_{w}^{1}(G)$ and $f \in$ $\mathscr{L}_{g}^{B}(G)$ be given. Then

$$
\begin{aligned}
(h * f)^{g}(x) & =\left\|\left(L_{x} g\right) \cdot(h * f)\right\|_{\infty} \leqq \int_{G}|h(y)|\left\|\left(L_{y^{-1}-1} g\right) \cdot f\right\|_{\infty} d y \\
& =\int|h(y)| L_{y}\left(f^{g}\right)(x) d y=|h| * f^{g}(x)
\end{aligned}
$$


holds and hence $\|h * f\|_{(B)} \leqq\|h\|_{1, W}\|f\|_{(B)}$.

Moreover, we shall use the following uniform partition of unity that was introduced by H. G. Feichtinger in [8] in a more general form. Let $V=V^{-1}$ be an open, relatively compact subset of $G$. Then there exists a subset $Y=\left\{y_{i}\right\}_{i \in I} \subset G$ such that

$$
G=\bigcup_{i \in I} y_{i} V \text { and } \sup _{x \in G}\left|\left\{i \mid x \in y_{i} V\right\}\right| \leqq C<\infty
$$

holds. Furthermore, there exists a bounded partition of unity $\left(\varphi_{i}\right)_{i \in I} \subset$ $\mathscr{C}^{\circ}(G)$, i.e.,

$$
\sum_{i \S I} \varphi_{i}(x)=1 \text { and } \sup _{i \in I}\left\|\varphi_{i}\right\|_{\infty} \leqq C^{\prime}<\infty,
$$

such that $\operatorname{supp} \varphi_{i} \subset y_{i} V, i \in I$.

REMARK 2.1. (i) The spaces $\mathfrak{B}^{p}(G)$ where introduced in [2] and the spaces $\mathscr{M}_{g}^{p}(G)$ in [12]. In [12] the independence of the spaces $\mathscr{C}_{g}^{p}(G)$ of the function $g$ was claimed but the argument used there fails, if no additional properties-related to commutativity-are imposed on $G$. In Theorem 2.4 we shall give necessary and sufficient conditions for the independence of $g$. In the case $B(G)=L^{p}(G)$ it turns out that the spaces $\mathscr{K}_{g}^{p}(G)$ are in fact independent of the choice of the function $g$ for arbitrary $G$. Moreover, this theorem shows that $\mathfrak{B}^{B}(G)=\mathscr{A}_{g}^{B}(G)$ if and only if these conditions are satisfied. Furthermore there will be given an example of a Banach space satisfying (B1)-(B3) such that $\mathscr{L}_{g}^{B}(G)$ is not independent of $g$.

(ii) In [2] it was shown that $\mathfrak{B}^{1}(G)=W^{1}(G)$ holds, where $W^{1}(G)$ denotes Wiener's algebra as introduced by $H$. G. Feichtinger in [5] and, using the main result of [5], $\mathfrak{B}^{p}(G)=\mathscr{K}_{g}^{p}(G)$ was derived. Our main theorem gives a direct proof of this result, as well. It seems to be worth noticing that it is possible to give a characterization of the Banach space $\mathscr{M}_{g}^{B}(G)$ in terms of functions of translation type without the direct use of a norm.

(iii) The spaces $\mathscr{L}_{g}^{p}(G)$ were considered by various authors using other but equivalent definitions (cf. [1], [4], [5], [10], [15]). Using the terminology of these authors we have successively: $\mathscr{A}_{g}^{p}(G)=$ $l^{p}(\mathscr{C}), \mathscr{H}_{g}^{p}(G)=\mathfrak{I}_{p}(G) \cap \mathscr{C}(G), \mathscr{H}_{g}{ }^{1}(G)=W^{1}(G), \mathscr{H}_{g}^{p}(\boldsymbol{R})=\left(\mathscr{C}^{\circ}, l^{p}\right)$ and $\mathscr{L}_{g}^{p}=\left(\mathscr{C}^{\circ}, l^{p}\right)$. In all these cases except in [5] $G$ is supposed to be abelian.

LEMMA 2.2. Let $V \subset G$ and $\left\{y_{i}\right\}_{i \in I} \subset G$ be given such that (*) holds and let $\left(\varphi_{i}\right)_{i \in I}$ be a partition of unity corresponding to the covering $\left(y_{i} V\right)$. Then each $\omega \in V^{1}(G)$ satisfies 


$$
\sum_{i \in I}\left\|\varphi_{i} \omega\right\|_{\infty} \leqq C \cdot \sum_{i \in I} \omega\left(y_{i}\right)<\infty
$$

Proof. $\left\|\varphi_{i} \omega\right\|_{\infty}=\sup _{x \in V}\left|\varphi_{i}\left(y_{i} x\right) \omega\left(y_{i} x\right)\right| \leqq\left\|\varphi_{i}\right\|_{\infty} C_{V} \omega\left(y_{i}\right)=C \cdot \omega\left(y_{i}\right)$ and therefore $\sum_{i \in I}\left\|\varphi_{i} \omega\right\|_{\infty} \leqq C \cdot \sum_{i \in I} \omega\left(y_{i}\right)$. Furthermore $\sup _{x \in G} \mid\{i \mid x \in$ $\left.y_{i} V\right\} \mid \leqq C^{\prime}$ implies $\sum_{i \in I} \Phi_{y_{i} V} \leqq C^{\prime}$ and hence

$$
\sum_{i \in I} \int_{y_{i} V} f(x) d x \leqq C^{\prime} \cdot \int_{G} f(x) d x
$$

for each $f \in L_{+}^{1}(G)$.

From this relation we derive:

$$
\sum_{i \in I} \omega\left(y_{i}\right) \leqq C_{V} \cdot \sum_{i \in I} \int_{y_{i} V} \omega(x) d x \leqq C_{V} C^{\prime} \cdot \int_{G} \omega(x) d x<\infty .
$$

Lemma 2.3. For all $g \in \mathscr{\mathscr { C }}(G), g \neq 0$, we have $\mathfrak{B}^{B}(G) \subset \mathscr{A l}_{g}^{B}(G)$.

Proof. Let $f \in \mathfrak{B}^{B}(G)$ with $|f| \leqq \omega$ and $\omega \in V^{B}(G)$. Then $\omega^{g}(x)=$ $\left\|\left(L_{x} g\right) \cdot \omega\right\|_{\infty}=\sup \{|g(y) \omega(x y)|, y \in \operatorname{supp} g\} \leqq C_{K}\|g\|_{\infty} \omega(x)$ and $\|f\|_{(B)} \leqq$ $\|\omega\|_{(B)} \leqq C\|\omega\|_{B}<\infty$ since $B(G)$ and $\mathscr{L}_{g}^{B}(G)$ satisfy (B2).

Now we are able to prove our main theorem.

THEOREM 2.4. The following conditions are equivalent.

(1) $R_{a} B^{g} \subset B$ and $\rho(a):=\sup \left\{\|\left(R_{a} f^{g}\left\|_{B} /\right\| f^{g} \|_{B}\right), f \in \mathscr{M}_{g}^{B}(G)\right\}$ is locally bounded for all $g \in \mathscr{K}(G), g \neq 0$.

(2) $\mathfrak{B}^{B}(G)=\mathscr{L}_{g}^{B}(G)$ for all $g \in \mathscr{K}(G), g \neq 0$.

(3) $\mathscr{M}_{g}{ }^{B}(G)$ is independent of the choice of the function $g \in$ $\mathscr{\%}(G), g \neq 0$, and different functions $g$ yield equivalent norms.

Proof. $\quad(1) \Rightarrow(2)$ We have to show that to every $f \in \mathscr{M}_{g}^{B}(G)$ there exists some $\omega_{f} \in V^{B}(G)$ with $|f| \leqq C \cdot \omega_{f}, C<\infty$.

Since $\rho$ is locally bounded, strictly positive and satisfies $\rho(x y) \leqq$ $\rho(x) \rho(y)$ we can choose functions $\omega, \omega_{1} \in V_{T}^{1}(G)$ such that $\omega \cdot \rho \leqq \omega_{1}$ holds (Lemma 1.2). If we now put:

$$
\omega_{f}(x):=\left\|\left(L_{x} \omega\right) \cdot f\right\|_{\infty}
$$

we obtain:

(i ) $|f(x)|=C \cdot\left|L_{x} \omega(x) f(x)\right| \leqq C \cdot \omega_{f}(x)$.

(ii) $\omega_{f}$ is continuous and $\omega_{f} \geqq 0$.

(iii) $\omega_{f}(x a)=\left\|\left(L_{x a} \omega\right) \cdot f\right\|_{\infty}=\sup _{y \in G}\left|\omega\left(a^{-1} x^{-1} y\right) f(y)\right|$

$$
\leqq \sup _{y \in G}\left|\omega\left(x^{-1} y\right) f(y)\right| \cdot C_{K}=C_{K} \cdot \omega_{f}(x) \text { for all } x \in G \text {. }
$$

(iv) Without loss of generality we can assume that supp $g$ contains an open, symmetric neighbourhood $V$ of $e$ satisfying $g(v) \cdot C^{\prime} \geqq 1$ for all $v \in V$. Now choose a set $\left\{y_{i}\right\}_{i \in I} \subset G$ such that $(*)$ is fullfilled 
and a corresponding partition of unity. Then we have:

$$
\begin{aligned}
\omega_{f}(x) & =\left\|\left(L_{x}\left(\sum_{i \in I} \varphi_{i} \omega\right)\right) \cdot f\right\|_{\infty} \leqq \sum_{i \in I} \|\left(L_{x}\left(\varphi_{i} \omega\right) \cdot f \|_{\infty}\right. \\
& \leqq C^{\prime} \cdot \sum_{i \in I}\left\|\left(L_{x}\left(\varphi_{i} \omega\right)\right) \cdot L_{x y_{2}} g \cdot f\right\|_{\infty} \leqq C^{\prime} \sum_{i \in I}\left\|\varphi_{i} \omega\right\|_{\infty}\left\|\left(L_{x y_{i}} g\right) \cdot f\right\|_{\infty} \\
& =C^{\prime} \sum_{i \in I}\left\|\varphi_{i} \omega\right\|_{\infty}\left(R_{y_{i}}\left(f^{g}\right)\right)(x) .
\end{aligned}
$$

Now Lemma 1.2 and Lemma 2.2 yield:

$$
\begin{aligned}
\left\|\omega_{f}\right\|_{B} & \leqq C^{\prime} \sum_{i \in I}\left\|\varphi_{i} \omega\right\|_{\infty}\left\|R_{y_{i}}\left(f^{g}\right)\right\|_{B} \leqq C^{\prime \prime} \sum_{i=1} \omega\left(y_{i}\right) \rho\left(y_{i}\right)\left\|f^{g}\right\|_{B} \\
& \leqq C^{\prime \prime} \sum_{i \in I} \omega_{1}\left(y_{i}\right)\|f\|_{(B)}<\infty
\end{aligned}
$$

Hence $\omega_{f} \in V^{B}(G)$ and $|f| \leqq \omega_{f}$.

$(2) \Rightarrow(3)$ is a simple application of the closed graph theorem.

$(3) \Rightarrow(1)$ Let $g \in \mathscr{K}(\mathrm{G})$ be given and $K \subset G$ be compact. Then there exists $h \in \mathscr{Y}_{+}(G)$ such that $\sup _{a \in K}\left|L_{a} g\right| \leqq h$ and $\left\|f^{h}\right\|_{B} \leqq$ $C \cdot\left\|f^{g}\right\|_{B}$ for all $f \in \mathscr{C}_{g}{ }^{B}(G)=\mathscr{C}_{h}^{B}(G), C<\infty$. Thus we obtain $\sup _{a \in K}\left\|R_{a}\left(f^{g}\right)\right\|_{B} \leqq C \cdot\left\|f^{g}\right\|_{B}$ for all $f \in \mathscr{C}_{g}^{B}(G)$ and hence $\rho$ is locally bounded.

From now on we shall always assume that the conditions of the above theorem are satisfied and therefore we shall write $\mathscr{C l}^{I B}(G)$ instead of $\mathscr{R}_{g}^{B}(G)$.

COROLlary 2.5. $\quad \mathbb{S}^{n}(G)$ is a Banach space with the norm \|\|$_{(B)}$ and satisfies the conditions (B1)-(B3).

REMARK 2.2. The following statements are easily derived from Theorem 2.4 .

(i) If $h \in \mathfrak{V}_{T}^{1}(G)$ then we have $\mathscr{C}^{B}(G)=\left\{f \in \mathscr{C}(G) \mid f^{h} \in B(G)\right\}$.

(ii) If $\mathscr{C}^{B_{2}} \subset B_{1}(G) \subset B_{2}(G)$ then $\mathscr{C}^{B_{2}}(G)=\mathscr{C l}^{B_{1}}(G)$.

(iii) The spaces $l^{p}\left(L^{q}\right)$ of measurable functions being locally in $L^{4}$ and globally in $l^{p}$ (cf. [1], [10], [15]) satisfy of course the conditions of our theorem. Especially $l^{p}\left(\mathscr{C}^{\circ}\right)=. \mathscr{C}^{p}$ holds (cf. [1]). It is easy to see that also $\mathscr{C l}^{p}=\mathscr{A}^{l^{p}\left(l^{q}\right)}$ holds.

At the end of this section we shall introduce a new family of Banach spaces satisfying (B1)-(B3) that will give us an example of a Banach space that does not satisfy the conditions of Theorem 2.3.

For a continuous function $f$ on $G$ and a closed subgroup $H<G$ $f / H$ shall denote the restriction from $f$ to $H$. For simplicity we write $\|f / H\|_{1}$ instead of $\|f / H\|_{L^{1}(I)}$.

Definition 2.2. Let $1 \leqq p \leqq \infty$. Then we define 


$$
E^{p}(G, H):=\left\{f \in L^{p}(G) \cap \mathscr{C}^{\circ}(G) \mid \sup _{x \in G}\left\|\left(L_{x} f\right) / H\right\|_{1}<\infty\right\} .
$$

TheOREM 2.6. $E^{p}(G, H)$ is a Banach space with the norm

$$
\|f\|_{E, p}:=\|f\|_{p}+\|f\|_{\infty}+\sup _{x \in \mathscr{G}}\left\|\left(L_{x} f\right) / H\right\|_{1},
$$

satisfying (B1)-(B3) with respect to $L^{1}(G)$.

Proof. It is a matter of routine to check that $E^{p}(G, H)$ is a Banach space and satisfies (B1) and (B2). Moreover, even $\left\|L_{y} f\right\|_{E, p}=$ $\|f\|_{E, p}$ holds. Now let $f \in L^{1}(G)$ and $g \in E^{p}(G, H)$ be given. Then we have

$$
\begin{aligned}
\left\|\left(L_{x}(f * g)\right) / H\right\|_{1} & \leqq \int_{I I} \int_{G}\left|L_{x} f(y)\right|\left|g\left(y^{-1} h\right)\right| d y d h \\
& =\int_{G}\left|L_{x} f(y)\right| \int_{H}\left|g\left(y^{-1} h\right)\right| d h d y \leqq\|f\|_{1} \cdot \sup _{y \in G}\left\|\left(L_{y} g\right) / H\right\|_{1}
\end{aligned}
$$

and hence $\|f * g\|_{E, p} \leqq\|f\|_{1} \cdot\|g\|_{E, p}$.

EXAMPle. Let us consider the space $E^{\infty}(G, H)$, where $G$ denotes the " $a x+b$ "-group, i.e., $G=\{(a, b) \mid a, b \in \boldsymbol{R}, a>0\}$ with multiplication $(a, b) \cdot\left(a^{\prime}, b^{\prime}\right):=\left(a a^{\prime}, a b^{\prime}+b\right)$ and $H:=\left\{\left(d^{n}, 0\right) \mid n \in Z, d\right.$ fixed $\}$, where $d$ will be chosen in a appropriate way. Then $H$ is a non-normal subgroup of $H$.

Now let $g, k \in \mathscr{K}_{+}(G)$ be given such that $g(e) \neq 0$ and $k(e) \neq 0$, and let $K_{1}:=\operatorname{supp} g$ and $K_{2}:=\operatorname{supp} k$. If furthermore, $c=\left(c_{1}, c_{2}\right)$, $c_{2} \neq 0$, is given and $c_{n}:=\left(d^{n}, 0\right) \cdot\left(c_{1}, c_{2}\right)$ then a simple computation shows that $d$ can be chosen such that

(*) $\quad K_{2} c_{n} K_{1}^{-1} H \cap K_{2} c_{m} K_{1}^{-1} H=\varnothing$ holds for all $n, m \geqq 1, n \neq m$.

Now define $f(x):=\sum_{n \geqq 1}(1 / n) R_{c_{n}^{-1}} k(x)$. Then we have

$$
f^{g}(x)=\left\|\left(L_{x} g\right) \cdot f\right\|_{\infty}=\sum_{n \geqq 1} \frac{1}{n}\left\|\left(L_{x} g\right) \cdot R_{c_{n}^{-1}} k\right\|_{\infty}
$$

due to $(*)$ since $\operatorname{supp}\left(f^{g}\right)=\bigcup_{n \geqq 1} K_{2} c_{n} K_{1}^{-1}$ and hence $f, f^{g} \in \mathscr{C}^{\circ}(G)$. Moreover (*) implies

$$
\begin{aligned}
\left\|\left(L_{x} f\right) / H\right\|_{1} & \leqq g(e)^{-1}\left\|\left(L_{x}\left(f^{g}\right)\right) / H\right\|_{1} \leqq g(e)^{-1} \sup _{\substack{n \geq 1 \\
h \in H}} \frac{1}{n}\left\|\left(L_{x^{-1} h} g\right) \cdot\left(R_{c_{n}^{-1}} k\right)\right\|_{\infty} \\
& \leqq g(e)^{-1}\|g\|_{\infty}\|k\|_{\infty} \text { for all } x \in G .
\end{aligned}
$$

Therefore we have $f, f^{g} \in E^{\infty}(G, H)$. On the other hand we have: 


$$
\begin{aligned}
\left\|\left(R_{c}\left(f^{g}\right)\right) / H\right\|_{1} & \geqq \sum_{n \geqq 1} f^{g}\left(c_{n}\right)=\sum_{n \geq 1} \frac{1}{n}\left\|\left(L_{c_{n}} g\right) \cdot\left(R_{c_{n}^{-1}} k\right)\right\|_{\infty} \\
& \geqq \sum_{n \geqq 1} \frac{1}{n}|g(e)||k(e)|=\infty \\
& \text { since }\left\{\left(d^{n}, 0\right), n \geqq 1\right\} \subset H \cap \operatorname{supp}\left(R_{c}\left(f^{g}\right)\right) \text { and } g(e), k(e) \neq 0 .
\end{aligned}
$$

Thus $f$ and $f^{g}$ are functions in $E^{\infty}(G, H)$ such that $R_{c}\left(f^{g}\right)$ is not in $E^{\infty}(G, H)$ and hence the space $E^{\infty}(G, H)$ does not satisfy the conditions (1)-(3) of Theorem 2.4 .

\section{Applications.}

THEOREM 3.1. $\mathfrak{B}^{1, w}(G)$ is the minimal space in the family of all Banach spaces satisfying (B1)-(B3) with respect to $L_{w}^{1}(G)$.

Proof. Let $f \in \mathfrak{B}^{1, w}(G)$ be given. By Remark 1.2 (resp. Lemma 1.4) $V^{1, w}(G)$ is dense in $\left(L_{w}^{1}\right)_{+}(G)$ and hence, given $\varepsilon>0$, there exists $\omega \in V^{1, w}(G)$ such that $|f| \leqq \omega$ and $\|\omega\|_{1, w} \leqq\|f\|_{1, w}+\varepsilon$. Choosing any $k \in \mathscr{K}_{+}(G)$ such that supp $k$ is contained in the component of the identity (which in turn is contained in $G(\omega)$ ), Lemma 1.1 yields $\omega \leqq$ $C_{k} \cdot\left(\omega^{*} k\right)$ and $C_{k}$ does not depend on $\omega$. Thus we get

$$
\|f\|_{B} \leqq\|\omega\|_{B} \leqq C_{k}\|k\|_{B}\|\omega\|_{1, w} \leqq C_{k}^{\prime}\left(\|f\|_{1, w}+\varepsilon\right) \text { for all } \varepsilon>0 .
$$

Hence $\|f\|_{B} \leqq C \cdot\|f\|_{1, w}$ for every $f \in \mathfrak{B}^{1, w}(G)$.

REMark 3.1. This theorem reduces to Corollary 3 of [2] and to Theorem 4 of [5] in the case $w \equiv 1$. The case $w \equiv 1$ was also generalized in an other direction in [8].

For the rest of this section we shall assume that $G$ is abelian and that $B(G)$ satisfies (B1)-(B3) with respect to $L^{1}(G)$.

THEOREM 3.2. Let $f \in L^{1}(G)$ with compact support be given. If $\hat{f} \in B(G)$ then $\hat{f} \in \mathscr{C}^{B}(G)$ and there exists a constant $M$ depending only on $B(G)$ and supp $f$ such that $\|\hat{f}\|_{(B)} \leqq M\|\hat{f}\|_{B}$.

Proof. Take any function $k \in \mathscr{K}_{+}(G)$ such that $k=1$ on supp $f$ and $\hat{k} \in \mathscr{L}^{1}(\hat{G})=\mathfrak{B}^{1}(\hat{G})$ (in fact for every function $k \in \mathscr{K}_{+}(G)$ with $\hat{k} \in L^{1}(\hat{G})$ one has $\hat{k} \in \mathfrak{B}^{1}(G)$, since $\mathfrak{B}^{1}(\hat{G})$ is a Segal algebra, cf. [2], [13]). Thus there is a function $\omega \in V^{1}(\hat{G})$ with $|\hat{k}| \leqq \omega$ and from $f=f \cdot k$ we derive $|\hat{f}| \leqq|\hat{f}| * \omega$. Since it is obvious that $|\hat{f}| * \omega \in V^{B}(\widehat{G})$ we have $\hat{f} \in \mathfrak{B}^{B}(\hat{G})$. Moreover, $(\hat{f})^{g} \leqq|\hat{f}| * \omega^{g}$ holds (see proof of Theorem 2.1) and hence $\|\hat{f}\|_{(B)}=\left\|(\hat{f})^{g}\right\|_{B} \leqq\left\|\omega^{g}\right\|_{1}\|\hat{f}\|_{B}=M\|\hat{f}\|_{B}$ with $M=\left\|\omega^{g}\right\|_{1}<$ $\infty$ since $\omega^{g} \sim \omega$ (see proof of Lemma 2.3). 
REMARK 3.2. (i) This theorem generalizes earlier results of F. Holland (cf. [10]), Edwards-Hewitt-Ritter (cf. [4]) and J. Stewart (cf. [15]). Besides, our proof seems to be the simplest one. For arbitrary abelian $G$ this theorem was first established by EdwardsHewitt-Ritter in the case $B(G)=L^{p}(G), 2 \leqq p<\infty$, in order to prove their main results on multipliers. But their proof, using the theory of entire functions, runs over several pages. The proofs of Holland $(G=\boldsymbol{R})$ and Stewart ( $G$ abelian) use Hölder's inequality together with other results from Harmonic Analysis.

(ii) Let $f$ satisfy the hypothesis of Theorem 3.2 with $B=l^{p}\left(L^{q}\right)$ (cf. [1]). Under these assumptions $\hat{f} \in l^{p}\left(L^{1}\right)$ is stated in Lemma 2 of [1]. Using Theorem 3.2 together with Remark 2.2 (iii) we get $\hat{f} \in \mathscr{C}^{p}(G)=l^{p}(\mathscr{C})$. This improves part of this lemma, since $l^{p}(\mathscr{C}) \varsubsetneqq$ $l^{p}\left(L^{1}\right)$ (except for discrete $G$ ).

Added in proof. In the paper "Banach convolution algebras of Wiener's type" (to appear in Proc. Conf. "Functions, Series, Operators", Budapest) H. G. Feichtinger introduced so called Banach spaces of Wiener's type $W(B, C)$. The construction involves a certain compact set $Q \subset G$. In order to show the independence of $Q$ the author assumes $C$ to be right invariant. Since it can be shown that for the special case $B=\mathscr{C}^{\circ}(G)$ this independence is equivalent to the independence of the spaces $\mathscr{C}_{g}{ }^{C}(G)$ of the function $g$, it follows from our example that one cannot omit a condition related to right invariance of $C$. Right invariance of $C$ also implies $\mathfrak{B}^{C}(G)=\mathscr{H}^{C}(G)=W\left(\mathscr{C}^{\circ}(G), C\right)$. In fact even for arbitrary spaces $W(B, C)$ our condition (i) of Theorem 2.4 , if modified in the obvious way, is necessary and sufficient for the independence of $Q$. However, a characterization of the spaces $W(B, C)$ in the sense of Theorem 2.4 by functions of translation type is impossible for general $B$.

\section{REFERENCES}

1. J. P. Bertrandias and C. Dupius, Transformation de Fourier sur les espace $l^{p}\left(L^{p^{\prime}}\right)$. Ann. Inst. Fourier, 29 (1979).

2. R. Bürger, Functions of translation type and Wiener's algebra, Arch. Math., 36 (1981), 73-78.

3. - Functions of translation type and functorial properties of Segal algebras I, Monatsh. Math., 90 (1980), 101-115.

4. R. E. Edwards, E. Hewitt and G. Ritter, Fourier multipliers for certain spaces of functions with compact supports, Inv. Math., 40 (1977), 37-57.

5. H. G. Feichtinger, A characterization of Wiener's algebra on locally compact groups, Arch. Math., 29 (1977), 136-140.

6. - On a class of convolution algebras of functions, Ann. Inst. Fourier, 27/3, (1977), 135-162.

7. Multipliers of Banach spaces of functions on groups, Math. Z., 152 (1976), $47-58$. 
8. H. G. Feichtinger, A characterization of minimal homogeneous Banach spaces, Proc. AMS, 81 (1981), 55-61.

9. G. I. Gaudry, Multipliers of weighted Lebesgue and measure spaces, Proc. London Math. Soc., (3), 19 (1969), 327-340.

10. F. Holland, Harmonic analysis on amalgams of $L^{p}$ and $l^{q}$, J. London Math. Soc., (2) 10 (1975), 295-305.

11. J. W. Jenkins, Growth of connected locally compact groups, J. Functional Analysis, 12 (1973), 113-127.

12. T. S. Liu, A. Rooij van and J. K. Wang, On some group algebra modules related to Wiener's algebra $M_{1}$, Pacific J. Math., 55 (1974), 507-520.

13. H. Reiter, Classical Harmonic Analysis and Locally Compact Groups, Oxford Univ. Press, 1968.

14. - Über den Satz von Weil-Cartier, Monatsh. Math., 86 (1978), 13-62.

15. J. Stewart, Fourier transforms of unbounded measures, Canad. J. Math., 31 (1979), 1281-1293.

Received August 5, 1980 and in revised form March 26, 1981.

UNIVERSITÄT WIEN

Strudlhofgasse 4

A 1090 Wien, Austria 



\section{PACIFIC JOURNAL OF MATHEMATICS}

\section{EDITORS}

DONALD BABBITT (Managing Editor)

University of California

Los Angeles, CA 90024

Hugo RossI

University of Utah

Salt Lake City, UT 84112

C. C. Moore and Arthur Agus

University of California

Berkeley, CA 94720
J. DugundJI

Department of Mathematics

University of Southern California

Los Angeles, CA 90007

R. FinN and J. MiLgRAM

Stanford University

Stanford, CA 94305

\section{ASSOCIATE EDITORS}
R. ARENS
E. F. BECKENBACH
B. H. NeumanN
F. WOLF
K. YoSHIDA

\section{SUPPORTING INSTITUTIONS}

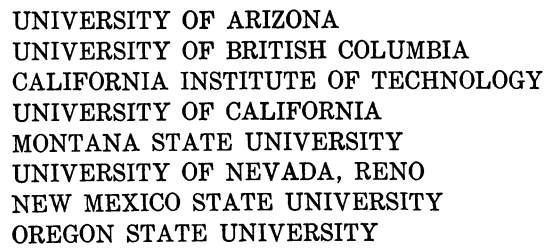

UNIVERSITY OF ARIZONA

UNIVERSITY OF BRITISH COLUMBIA CALIFORNIA INSTITUTE OF TECHNOLOGY UNIVERSITY OF CALIFORNIA MONTANA STATE UNIVERSITY

UNIVERSITY OF NEVADA, RENO NEW MEXICO STATE UNIVERSITY OREGON STATE UNIVERSITY

\author{
UNIVERSITY OF OREGON \\ UNIVERSITY OF SOUTHERN CALIFORNIA \\ STANFORD UNIVERSITY \\ UNIVERSITY OF AAWAII \\ UNIVERSITY OF TOKYO \\ UNIVERSITY OF UTAH \\ WASHINGTON STATE UNIVERSITY \\ UNIVERSITY OF WASHINGTON
}

The Supporting Institutions listed above contribute to the cost of publication of this Journal, but they are not owners or publishers and have no responsibility for its content or policies,

Mathematical parers intended for publication in the Pacific Journal of Mathematics should be in typed form or offset-reproduced, (not dittoed), double spaced with large margins. Please do not use built up fractions in the text of the manuscript. However, you may use them in the displayed equations. Underline Greek letters in red, German in green, and script in blue. The first paragraph or two must be capable of being used separately as a synopsis of the entire paper. Please propose a heading for the odd unmbered pages of less than 35 characters. Manuscripts, in triplicate, may be sent to any one of the editors. Please classify according to the scheme of Math. Reviews, Index to Vol. 39. Supply name and address of author to whom proofs should be sent. All other communications should be addressed to the managing editor, or Elaine Barth, University of California, Los Angeles, California, 90024 .

50 reprints to each author are provided free for each article, only if page charges have been substantially paid. Additional copies may be obtained at cost in multiples of 50 .

The Pacific Journal of Mathematics is issued monthly as of January 1966, Regular subscription rate: $\$ 114.00$ a year (6 Vol., 12 issues). Special rate: $\$ 57.00$ a year to individual members of supporting institution.

Subscriptions, orders for numbers issued in the last three calendar years, and changes of address shoud be sent to Pacific Journal of Mathematics, P.O. Box 969, Carmel Valley, CA 93924, U.S.A. Old back numbers obtainable from Kraus Periodicals Co., Route 100, Millwood, NY 10546.

\section{PUBLISHED BY PACIFIC JOURNAL OF MATHEMATICS, A NON-PROFIT CORPORATION}

Printed at Kokusai Bunken Insatsusha (International Academic Printing Co., Ltd.). 8-8, 3-chome, Takadanobaba, Shinjuku-ku, Tokyo 160, Japan.

Copyright (C) 1982 by Pacific Journal of Mathematics Manufactured and first issued in Japan 


\section{Pacific Journal of Mathematics}

Vol. 100, No. $2 \quad$ October, 1982

Kenneth F. Andersen, On the transformation of Fourier coefficients of

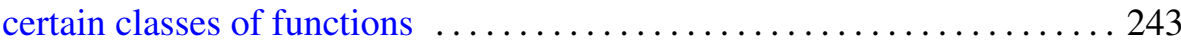

Steven Albert Bleiler, Realizing concordant polynomials with prime knots

Reinhard Bürger, Functions of translation type and solid Banach spaces of functions

Ulrich Daepp, The saturation of $k$-analytic rings and topological equivalence of associated analytic set germs .................. 271

Persi W. Diaconis and David Amiel Freedman, On the maximum difference between the empirical and expected histograms for sums . . . 287

David Amiel Freedman, On the maximum of scaled multinomial

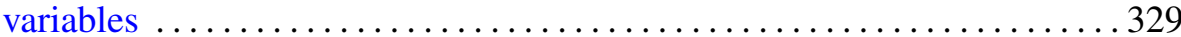

Persi W. Diaconis and David Amiel Freedman, On the difference between the empirical histogram and the normal curve, for sums. II ......... 359

Persi W. Diaconis and David Amiel Freedman, On the mode of an

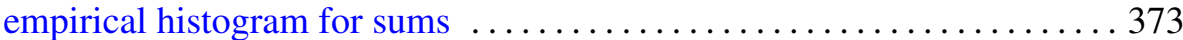

Jutta Hausen, Supplemented modules over Dedekind domains 387

Elyahu Katz, A moduli representation for the classification of twisted tensor

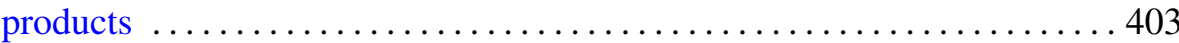

H. C. Madhekar and N. K. Thakare, Biorthogonal polynomials suggested by the Jacobi polynomials

Ted R. Pettis, Collections of covers of metric spaces 425

Ryōtarō Satō, Maximal functions for a semiflow in an infinite measure space

Michael Jay Stob, Invariance of properties under automorphisms of the lattice of recursively enumerable sets 